Chronic Obstructive Pulmonary Diseases:

Journal of the COPD Foundation

COPD

FOUNDATION

\author{
Journal Club
}

\title{
Journal Club- COPD Exacerbations and Gastroesophageal Reflux Disease: Why Proton Pump Inhibitor Therapy is Not Enough
}

\author{
Ron Balkissoon, MD, MSc, DIH, FRCPC ${ }^{1}$
}

Abbreviations: Global initiative for chronic Obstructive Lung Disease, GOLD; chronic obstructive pulmonary disease, COPD; COPD Genetic Epidemiology, COPDGene ${ }^{\oplus}$; gastroesophageal reflux disease, GERD; proton pump inhibitor, PPI; esophagogastric junction, EGJ; lower esophageal sphincter, LES; continuous positive airway pressure, CPAP; bilevel positive airway pressure, BiPAP

Citation: Balkissoon R. Journal club-COPD exacerbations and gastroesophageal reflux disease: why proton pump inhibitor therapy is not enough. Chronic Obstr Pulm Dis. 2019;6(4):374-379. doi: https://doi.org/10.15326/jcopdf.6.4.2019.0162

\section{Denver, Colorado}

\section{Address correspondence to:}

Ron Balkissoon, MD, MSc, DIH, FRCPC

balkissoonr@njhealth.org

\section{Keywords}

chronic obstructive pulmonary disease; COPD; exacerbations; gastroesophageal reflux disease; proton pump inhibitor therapy

\section{Introduction}

Exacerbations are associated with increased morbidity and mortality, accelerated rates of decline in lung function, poor quality of life and higher health care utilization. ${ }^{1}$ The Global initiative for chronic Obstructive Lung Disease (GOLD) guidelines have identified exacerbations in addition to symptoms as the 2 main "treatable traits" to target for our therapeutic strategies for patients with chronic obstructive pulmonary disease (COPD). ${ }^{1}$ The so called "frequent-exacerbation phenotype" remains poorly defined and characterized. ${ }^{2}$ It is likely this represents a heterogeneous population that may present with some combination of various characteristics that increase the risk of having exacerbations. Spiromics and COPD Genetic Epidemiology (COPDGene ${ }^{\circledR}$ ) studies that have examined the utility of blood biomarkers to predict exacerbations, did not add substantial predictive power to established clinical co-variates for exacerbations. ${ }^{3}$ Interestingly, studies from COPDGene ${ }^{\circledR}$ have identified several clinical characteristics that appear to infer an increased risk of exacerbations. While a history of previous exacerbations, (moderate or severe), appears to be the strongest predictor of future exacerbations, investigators have also identified a history of gastroesophageal reflux or heartburn, poorer quality of life, lower lung function and elevated white cell count. ${ }^{2}$ Indeed, COPDGene ${ }^{\circledast}$ investigators reported that the evidence of a hiatal hernia on computed tomography scans of the chest was associated with a higher risk of exacerbations. ${ }^{4}$ It has been presumed that this reflects the potential role of gastroesophageal reflux as a cause for frequent exacerbations. Aside from sleep apnea and stroke, gastroesophageal reflux disease (GERD) had the highest association with poorer quality of life of various comorbidities found in COPD patients. While it is true that previous studies have shown introduction of acid into the lower esophagus is capable of inducing bronchoconstriction, ${ }^{5-7}$ our experience and indeed many others is that a large proportion of patients continue to have recurrent exacerbations despite being on proton pump inhibitor (PPI) therapy. 4,8 Another hypothesis is that GERD is associated with proximal extension and silent aspiration. ${ }^{8}$ While PPI therapy may play a role in reducing the impact of gastroesophageal refluxate being aspirated in the lungs, even non-acid may be potentially damaging and contribute to frequent exacerbations. ${ }^{8}$ In fact, studies have reported an increased risk of pneumonia in patients treated with PPI therapy, however this may be more a reflection of an association (i.e., patients that have reflux are more likely to aspirate and develop pneumonia than a true cause and effect). Furthermore, many patients with COPD may have dysphasia and swallowing problems that could also contribute to 
recurrent aspiration and exacerbation of COPD. ${ }^{8,9}$ Further, it has been proposed that aspiration may contribute to the development of dysbiosis in COPD patients that may also be an important feature of the frequent exacerbation phenotype. ${ }^{10-13}$ GERD in COPD patients has been associated with the COPD/ bronchiectasis overlap. ${ }^{14-16}$ This may indeed be one of the common clinical scenarios (phenotypes) in which use of inhaled corticosteroids may be associated with an increased risk of pneumonia. ${ }^{10,17-20}$ The extent and mechanisms by which aspiration may contribute to frequent exacerbations remains poorly understood and is likely complex including factors that increase the risk of aspiration and factors that increase susceptibility including structural changes and alterations in local immunity. In this Journal Club we will review some of the more recent literature published regarding these issues.

Note: Abstracts are presented in their original, published format and have not been edited to match JCOPDF style.

\section{Abstract 1 Aspiration Pneumonia and Related Syndromes}

Lee AS, Ryu JH. Mayo Clin Proc. 2018;93(6):752-762. doi: https://doi.org/10.1016/j.mayocp.2018.03.011

Aspiration is a syndrome with variable respiratory manifestations that span acute, life-threatening illnesses, such as acute respiratory distress syndrome, to chronic, sometimes insidious, respiratory disorders such as aspiration bronchiolitis. Diagnostic testing is limited by the insensitivity of histologic testing, and although gastric biomarkers for aspiration are increasingly available, none have been clinically validated. The leading mechanism for microaspiration is thought to be gastroesophageal reflux disease, largely driven by the increased prevalence of gastroesophageal reflux across a variety of respiratory disorders, including chronic obstructive pulmonary disease, asthma, idiopathic pulmonary fibrosis, and chronic cough. Failure of therapies targeting gastric acidity in clinical trials, in addition to increasing concerns about both the overuse of and adverse events associated with proton pump inhibitors, raise questions about the precise mechanism and causal link between gastroesophageal reflux and respiratory disease. Our review summarizes key aspiration syndromes with a focus on reflux-mediated aspiration and highlights the need for additional mechanistic studies to find more effective therapies for aspiration syndromes.

\section{Comments}

This is an excellent review of the various ways in which aspiration may impact the lung both acutely and chronically. It provides a very nice overview of thoracoabdominal mechanics in terms of the ways in which the transdiaphragmatic pressure gradient interacts with the esophagogastric junction/lower esophageal sphincter (EGJ/LES) complex to promote reflux. It provides insights into why respiratory diseases may increase the propensity for COPD and asthma patients to develop reflux disease. There is also a very nice overview of the various defenses that will help to prevent reflux from occurring and leading to aspiration including the EGJ and LES, esophageal peristalsis, intact swallowing mechanisms, vocal folds, cough and mucocilliary function and local immune responses for most individuals. The article provides a useful framework to understand the complex balance between pro-reflux and pro-aspiration factors and the bodies various defense mechanisms and why a focus on PPI therapy to deal with this issue is likely to be unsuccessful in addressing a number of issues. The article points to variables such as the volume, pepsin content, bile acid content, and the element of gastric particulates that may impact the varying consequences of aspiration. Aside from examining these factors for patients with COPD and asthma, it also looks at acute aspiration syndromes, interstitial lung disease, chronic cough bronchiectasis and diffuse aspiration bronchiolitis. Finally, there is a very nice summary that takes all these potential contributing factors into consideration and outlines the variety of potential therapeutic interventions that should be considered ranging from acid suppression, promotility agents, speech therapy, cough suppression, bronchial hygiene and agents to reduce transient lower esophageal sphincter relaxations. 


\section{Abstract 2 \\ Oropharyngeal Swallow Physiology and Swallowing-Related Quality of Life in Underweight Patients With Concomitant Advanced Chronic Obstructive Pulmonary Disease}

Garand KL, Strange C, Paoletti L, Hopkins-Rossabi T, Martin-Harris B. Int J Chron Obstruct Pulmon Dis. 2018;13:2663-2671.

doi: https://doi.org/10.2147/COPD.S 165657

\section{BACKGROUND:}

Swallowing impairment (dysphagia) has been associated with COPD and may contribute to exacerbations of this chronic and progressive disease. Further, risk of mortality increases with concomitant presence of cachexia in the COPD population. The purpose of this prospective study was to depict oropharyngeal swallowing physiology in underweight patients with stable but advancedstage COPD.

\section{PATIENTS AND METHODS:}

Ten underweight patients with stable but advanced COPD underwent a modified barium swallow study. Analysis of oropharyngeal swallowing function was completed using the standardized Modified Barium Swallow Impairment Profile and the PenetrationAspiration Scale. Scores from the Dysphagia Handicap Index and 10-item Eating Assessment Tool were collected to assess patient perception of swallowing difficulty. Findings were compared to age- and sex-matched healthy controls.

\section{RESULTS:}

Significantly higher MBSImP oral total scores $(P=0.007)$ were observed in COPD patients compared to matched controls, but no difference was observed in pharyngeal total scores $(P=0.105)$. Patients with COPD had significantly higher maximum PAS scores compared with controls $(P=0.030)$. There was no significant difference in EAT-10 or DHI scores between patients with COPD and controls $(P=0.41$ and $P=0.08$, respectively).

\section{CONCLUSION:}

Underweight patients with severe but stable COPD present with dysphagia that may not be recognized by the patient. Further investigation is needed to elucidate the interaction between the respiratoryswallowing systems, how muscular weakness may contribute to swallowing impairment, and responsiveness to swallowing treatment.

\section{KEYWORDS:}

COPD; MBSImP; dysphagia; swallowing impairment; underweight

\section{Comments}

This is a small study, but it demonstrates the increased risk of aspiration in COPD patients and the fact that many COPD patients may not be aware of their increased risks of aspiration. It is reasonable to suggest that patients that demonstrate an increased risk of pneumonia should be assessed for aspiration with tailored barium studies even if they deny any increased risk of aspiration.

\section{Abstract 3 Effects of Noninvasive Ventilation on the Coordination Between Breathing and Swallowing in Patients With Chronic Obstructive Pulmonary Disease}

Hori R, Ishida R, Isaka M, Nakamura T, Oku Y. Int J Chron Obstruct Pulmon Dis. 2019;14:1485-1494.

doi: https://doi.org/10.2147/COPD.S205543

\section{PURPOSE:}

As shown in our previous study, inspiration after swallowing (SW-I) increases during the bi-level positive airway pressure ventilation (BiPAP) in healthy subjects because swallowing-associated noninspiratory flow (SNIF) triggers inspiratory support, while SW-I during continuous positive pressure ventilation (CPAP) is rare. In the present study, we evaluated the coordination between breathing and swallowing during spontaneous breathing, BiPAP, and 
CPAP in patients with chronic obstructive pulmonary disease (COPD).

\section{PATIENTS AND METHODS:}

This study is a prospective intervention study at the Hoshigaoka Medical Center (November 01, 2015-April 30, 2018). We simultaneously recorded the respiratory flow, laryngeal motion, and swallowing sounds during saliva swallowing in patients with COPD. We estimated the respiratory phase after swallowing, frequency of SNIF, the duration of the respiratory pause during swallowing, and timing of swallowing in the respiratory cycle and compared these parameters among control, CPAP, and BiPAP conditions.

\section{RESULTS:}

The expiration after swallowing (SW-E) frequency was associated with the occurrence of SNIF $(p<0.01)$, pause duration $\leq 0.8 \mathrm{~s} \quad(p<0.01)$, and timing of swallowing at the intermediate respiratory phase (50-80\% of the respiratory cycle from the onset of inspiration) $(p<0.01)$. In particular, the occurrence of SNIF most substantially affected the SW-E frequency. The SW-I frequencies under the control, CPAP, and BiPAP conditions were $35.0 \%, 3.0 \%$, and $37.7 \%$, respectively. The pause durations were shorter during CPAP and BiPAP than under the control condition $(p<0.01)$. During CPAP, the occurrence rates of SW-E. Residual denotes the percentage difference between observed and expected values (residual $=10.8$ : $p<0.01$ ) and SNIF (residual =9.1: $p<0.01$ ) were significantly increased, and timing of swallowing shifted toward the intermediate respiratory phase (residual=3.5: $p<0.01$ ).

\section{CONCLUSION:}

CPAP decreases the SW-I frequency, increases the SNIF occurrence, and normalizes the timing of swallowing, all of which suggest that CPAP alleviates the risk of aspiration in patients with COPD.

\section{KEYWORDS:}

chronic obstructive; continuous positive airway pressure; deglutition; pulmonary disease; respiration

\section{Comments}

While this is a very small study it would suggest that for patients who are at an increased risk of aspiration pneumonia or COPD exacerbations, continuous positive airway pressure (CPAP) ventilation may be a safer modality than bilevel positive airway pressure (BiPAP) (see below). Further studies are required.

\section{Abstract 4 The Mortality Risk Factor of Community Acquired Pneumonia Patients With Chronic Obstructive Pulmonary Disease: A Retrospective Cohort Study}

Dai RX, Kong QH, Mao B, et al. BMC Pulm Med. 2018;18(1):12.

doi: https://doi.org/10.1186/s12890-018-0587-7

\section{BACKGROUND:}

Chronic obstructive pulmonary disease (COPD) is one of the most common comorbidities in community acquired pneumonia (CAP) patients. We aimed to investigate the characteristics and mortality risk factors of COPD patients hospitalized with CAP.

\section{METHODS:}

A retrospective cohort study was conducted at Shanghai Pulmonary Hospital and Shanghai Dahua Hospital. Clinical and demographic data in patients diagnosed with CAP were collected between January 2015 and June 2016. Logistic regression analysis was performed to screen mortality risk factors of COPD patients hospitalized with CAP.

\section{RESULTS:}

Of the total 520 CAP patients, 230 (44.2\%) patients had been diagnosed comorbid with COPD (COPDCAP). CAP patients comorbid with COPD patients had higher rate of need for ICU admission (18.3\% vs $13.1 \%$ ) and need for NIMV (26.1\% vs $1.4 \%$ ) than without COPD (nCOPD-CAP). The PSI, CURB-65 and APACHE-II scores in COPD-CAP patients were higher than that in nCOPD-CAP patients (95 vs $79, \mathrm{P}<0.001$; 1 vs $1, \mathrm{P}<0.001 ; 13$ vs $8, \mathrm{P}<0.001$, respectively). Logistic regression analysis indicated that aspiration, D-dimer $>2.0 \mu \mathrm{g} / \mathrm{mL}$ and CURB-65 $\geq 3$ were risk factors associated with in-hospital mortality ((odd ratio) $\mathrm{OR}=5.678, \quad \mathrm{OR}=4.268, \quad \mathrm{OR}=20.764$, respectively) in COPD-CAP patients. The risk factors associated with 60-day mortality in COPD-CAP 
patients were comorbid with coronary heart disease, aspiration, need for NIMV (non-invasive mechanical ventilation) and $C U R B-65 \geq 3(\mathrm{OR}=5.206, \mathrm{OR}=7.921$, $\mathrm{OR}=3.974, \mathrm{OR}=18.002$, respectively).

\section{CONCLUSIONS:}

COPD patients hospitalized with CAP had higher rate of need for NIMV, need for ICU admission and severity scores than those without COPD. Aspiration, D-dimer $>2.0 \mathrm{\mu g} / \mathrm{mL}$, comorbid with coronary heart disease, need for NIMV and CURB-65 $\geq 3$ were mortality risk factors in CAP patients comorbid with COPD.

\section{Comments}

This study points out the high incidence/risk of pneumonia in COPD patients and the association with risk of aspiration. This is a retrospective study and aspiration risk was assessed by report in the chart and prospectively evaluated. This paper also brings into focus the relevance of the article above regarding differences between BiPAP and CPAP in terms of aspiration risk. It would appear that, if effective, CPAP may be a better option than BiPAP.

\section{Abstract 5 The Lung Tissue Microbiota of Mild and Moderate Chronic Obstructive Pulmonary Disease}

Pragman AA, Lyu T, Baller JA, et al. Microbiome. 2018;6(1):7.

doi: https://doi.org/10.1186/s40168-017-0381-4

\section{BACKGROUND:}

Oral taxa are often found in the chronic obstructive pulmonary disease (COPD) lung microbiota, but it is not clear if this is due to a physiologic process such as aspiration or experimental contamination at the time of specimen collection.

\section{METHODS:}

Microbiota samples were obtained from nine subjects with mild or moderate COPD by swabbing lung tissue and upper airway sites during lung lobectomy. Lung specimens were not contaminated with upper airway taxa since they were obtained surgically. The microbiota were analyzed with $16 \mathrm{~S}$ rRNA gene qPCR and $16 \mathrm{~S}$ rRNA gene hypervariable region 3 (V3) sequencing. Data analyses were performed using QIIME, SourceTracker, and R.

\section{RESULTS:}

Streptococcus was the most common genus in the oral, bronchial, and lung tissue samples, and multiple other taxa were present in both the upper and lower airways. Each subject's own bronchial and lung tissue microbiota were more similar to each other than were the bronchial and lung tissue microbiota of two different subjects (permutation test, $\mathrm{p}=0.0139$ ), indicating more within-subject similarity than between-subject similarity at these two lung sites. Principal coordinate analysis of all subject samples revealed clustering by anatomic sampling site (PERMANOVA, $p=0.001$ ), but not by subject. SourceTracker analysis found that the sources of the lung tissue microbiota were $21.1 \%$ (mean) oral microbiota, $8.7 \%$ nasal microbiota, and $70.1 \%$ unknown. An analysis using the neutral theory of community ecology revealed that the lung tissue microbiota closely reflects the bronchial, oral, and nasal microbiota (immigration parameter estimates $0.69,0.62$, and 0.74 , respectively), with some evidence of ecologic drift occurring in the lung tissue.

\section{CONCLUSION:}

This is the first study to evaluate the mild-moderate COPD lung tissue microbiota without potential for upper airway contamination of the lung samples. In our small study of subjects with COPD, we found oral and nasal bacteria in the lung tissue microbiota, confirming that aspiration is a source of the COPD lung microbiota.

\section{KEYWORDS:}

Emigration and immigration; Lung; Microbiota; Pulmonary disease, chronic obstructive; RNA, Ribosomal, 16S; Streptococcus

\section{Comments}

This study, while having a small number of patients, demonstrates contributions of oral and nasal microbiota aspiration to the microbiome of the lung. For patients that have significant laryngopharyngeal reflux it is indeed possible the GI refluxate may very well contribute to dysbiosis in the lung, but this requires further study. ${ }^{12,13}$ 


\section{References}

1. Global Initiative for Chronic Obstructive Pulmonary Disease (GOLD). Global strategy for the diagnosis, management, and prevention of chronic obstructive pulmonary disease, 2019. GOLD website. https://goldcopd.org/gold-reports/.

Published December 2018. Accessed October 2019.

2. Hurst J, Vestbo J, Anzueto A, et al. Susceptibility to exacerbation in chronic obstructive pulmonary disease. $N$ Engl J Med. 2010;363(12):1128-1138.

doi: https://doi.org/10.1056/NEJMoa0909883

3. Keene JD, Jacobson S, Kechris K, et al. Biomarkers predictive of exacerbations in the SPIROMICS and COPDGene cohorts. Am J Respir Crit Care Med. 2017;195(4):473-481.

doi: https://doi.org/10.1164/rccm.201607-13300C

4. Martinez $\mathrm{CH}$, Okajima Y, Murray S, et al. Impact of self-reported gastroesophageal reflux disease in subjects from COPDGene cohort. Respir Res. 2014;15:62.

doi: https://doi.org/10.1186/1465-9921-15-62

5. Amarasiri DL, Pathmeswaran A, Silva HJ, Ranasinha CD. Response of the airways and autonomic nervous system to acid perfusion of the esophagus in patients with asthma: a laboratory study. BMC Pulm Med. 2013;13:33.

doi: https://doi.org/10.1186/1471-2466-13-33

6. Harding SM, Schan CA Guzzo MR, Alexander RW, Bradley LA, Richter JE. Gastroesophageal reflux-induced bronchoconstriction. Is microaspiration a factor? Chest. 1995; 108(5):1220-1227.

doi: https://doi.org/10.1378/chest.108.5.1220

7. Wu DN, Tanifuji Y, Kobayashi H, et al. Effects of esophageal acid perfusion on airway hyperresponsiveness in patients with bronchial asthma. Chest. 2000;118(6): 1553-1556.

doi: https://doi.org/10.1378/chest.118.6.1553

8. Lee AS, Ryu JH. Aspiration pneumonia and related syndromes. Mayo Clin Proc. 2018;93(6):752-762.

doi: https://doi.org/10.1016/j.mayocp.2018.03.011

9. Terada K,Muro S, Ohara T, et al. Abnormal swallowing reflex and COPD exacerbations. Chest. 2010;137(2):326-332.

doi: https://doi.org/10.1378/chest.09-0482

$\overline{\text { 10. Singanayagam A, Glanville N, Cuthbertson L, et al. Inhaled }}$ corticosteroid suppression of cathelicidin drives dysbiosis and bacterial infection in chronic obstructive pulmonary disease. Sci Transl Med. 2019;11(507):eaav3879.

doi: https://doi.org/10.1126/scitranslmed.aav3879

11. Wang Z, Singh R, Miller BE, et al. Sputum microbiome temporal variability and dysbiosis in chronic obstructive pulmonary disease exacerbations: an analysis of the COPDMAP study. Thorax. 2018;73(4):331-338.

doi: https://doi.org/10.1136/thoraxjnl-2017-210741
12. Jette ME, Dill-McFarland KA, Hanshew AS, Suen G, Thibeault SL. The human laryngeal microbiome: effects of cigarette smoke and reflux. Sci Rep. 2016;6:35882.

doi: https://doi.org/10.1038/srep35882

13. Yang L, Chaudhary N, Baghdadi J, Pei Z. Microbiome in reflux disorders and esophageal adenocarcinoma. Cancer J. 2014;20(3): 207-210. doi: https://doi.org/10.1097/PPO.0000000000000044

14. Balkissoon R. Journal Club -bronchiectasis/COPD overlap: syndrome versus treatable trait? Chronic Obstr Pulm Dis. 2019; 6(2):193-199.

doi: https://doi.org/10.15326/jcopdf.6.2.2019.0134

15. Chung WS, Lin CL. Acute respiratory events in patients with bronchiectasis-COPD overlap syndrome: a population-based cohort study. Respir Med. 2018;140:6-10.

doi: https://doi.org/10.1016/j.rmed.2018.05.008

16. Polverino E, Dimakou K, Hurst J, et al. The overlap between bronchiectasis and chronic airway diseases: state of the art and future directions. Eur Respir J. 2018;52(3):1800328.

doi: https://doi.org/10.1183/13993003.00328-2018

17. Almagro P, Martinez-Camblor P, Soriano JB. Inhaled corticosteroids and pneumonia mortality in COPD patients. Eur Respir J. 2019; 54(3):1901035. doi: https://doi.org/10.1183/13993003.01035-2019

18. Janson C, Stratelis G, Miller-Larsson A, Harrison TW, Larsson K. Scientific rationale for the possible inhaled corticosteroid intraclass difference in the risk of pneumonia in COPD. Int $J$ Chron Obstruct Pulmon Dis. 2017;12:3055-3064.

doi: https://doi.org/10.2147/COPD.S143656

19. Tashkin DP, Miravitlles M, Celli BA, et al. Concomitant inhaled corticosteroid use and the risk of pneumonia in COPD: a matched-subgroup post hoc analysis of the UPLIFT(R) trial. Respir Res. 2018;19(1):196. doi: https://doi.org/10.1186/s12931-018-0874-0

20. Yang HH, Lai CC, Wang YH, et al. Severe exacerbation and pneumonia in COPD patients treated with fixed combinations of inhaled corticosteroid and long-acting beta2 agonist. Int $J$ Chron Obstruct Pulmon Dis. 2017;12:2477-2485. doi: https://doi.org/10.2147/COPD.S139035 